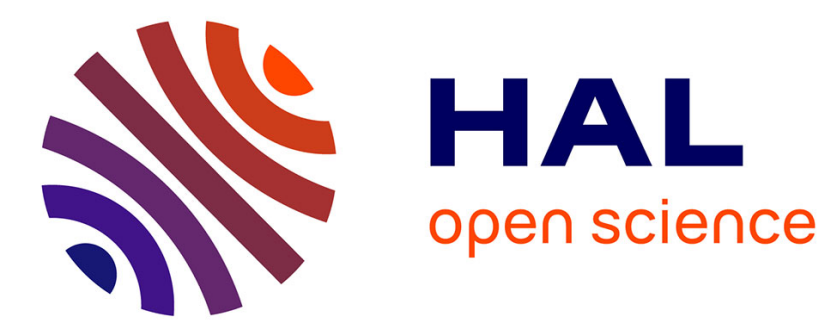

\title{
Two-dimensional numerical model of Marangoni surfers: From single swimmer to crystallization
}

Clément Gouiller, Christophe Ybert, Cécile Cottin-Bizonne, Florence Raynal, Mickaël Bourgoin, Romain Volk

\section{- To cite this version:}

Clément Gouiller, Christophe Ybert, Cécile Cottin-Bizonne, Florence Raynal, Mickaël Bourgoin, et al. Two-dimensional numerical model of Marangoni surfers: From single swimmer to crystallization. Physical Review E , 2021, 104 (6), pp.064608. 10.1103/physreve.104.064608 . hal-03491685

\section{HAL Id: hal-03491685 \\ https://hal.science/hal-03491685}

Submitted on 17 Dec 2021

HAL is a multi-disciplinary open access archive for the deposit and dissemination of scientific research documents, whether they are published or not. The documents may come from teaching and research institutions in France or abroad, or from public or private research centers.
L'archive ouverte pluridisciplinaire HAL, est destinée au dépôt et à la diffusion de documents scientifiques de niveau recherche, publiés ou non, émanant des établissements d'enseignement et de recherche français ou étrangers, des laboratoires publics ou privés. 


\title{
Two-dimensional numerical model of Marangoni surfers: From single swimmer to crystallization
}

\author{
Clément Gouiller, Christophe Ybert, and Cécile Cottin-Bizonne (1) \\ Institut Lumière Matière, Université de Lyon, Université Claude Bernard Lyon 1, CNRS, F-69622 Villeurbanne, France \\ Florence Raynal $\odot$ \\ Laboratoire de Mécanique des Fluides et d'Acoustique, Université de Lyon, Ecole Centrale de Lyon, Université Claude Bernard Lyon 1, \\ INSA Lyon, CNRS, F-69134 Écully, France \\ Mickaël Bourgoin and Romain Volk (1)* \\ Laboratoire de Physique, Université de Lyon, École Normale Supérieure de Lyon, CNRS, F-69342 Lyon, France
}

(Received 20 September 2021; accepted 23 November 2021; published 17 December 2021)

\begin{abstract}
We numerically study the dynamics of an ensemble of Marangoni surfers in a two-dimensional and unconfined space. The swimmers are modeled as Gaussian sources of surfactant generating surface tension gradients and are shown to follow the Marangoni flow filtered at their spatial scale in the lubrication regime, an unstable situation leading to spontaneous motion as soon as the Marangoni effect is intense enough. As the system is fully unconstrained, it is possible to study the various dynamical regimes from single swimmer, two-body interaction, to the many-particles case characterized by an efficient particle dispersion. We show that, although the present model is very simple, it reproduces the experimentally observed transition between a regime of dispersion by random agitation when the number of swimmers is moderate to the regime of crystallization with imperfect hexagonal lattice at high density.
\end{abstract}

DOI: 10.1103/PhysRevE.104.064608

\section{INTRODUCTION}

Active matter, made of a collection of self-propelled entities, has emerged over the past decades as a new class of out-of-equilibrium system with rich collective properties [1-7]. Inspired by animal, biological, or more synthetic selfpropelled objects, propulsion direction is usually hard-coded in an intrinsic asymmetry. Accordingly, the numerous and fruitful modelizations to date mostly considered a propulsion of fixed velocity or force whose direction is bound to the particle orientation and whose dynamics depends on external interaction [8-10].

However, the appealing possibility to have self-propelled particles without demanding the requirement of asymmetric fabrication emerged with swimming droplets or surfers that exhibit spontaneous symmetry breaking [11-13]. So far, studies on such active particles mostly addressed the swimming instability of isolated individuals $[14,15]$, or collective dynamics in one-dimensional (1D) geometries [16,17]. Far less is known concerning multiparticles dynamics in higher dimensions, although such a complex system, driven by long-range chemical interaction, can exhibit strongly fluctuating states [7] or crystallized states [12] depending on the number of swimmers per unit area. Yet, with the swimming direction not slaved to the particle orientation, one may expect these systems to escape generic flocking or motility-induced phase separation (MIPS) scenario [18,19] and define a new class of dynamics.

*Corresponding author: romain.volk@ens-lyon.fr
In the present article, we propose a two-dimensional (2D) model of symmetric active particles inspired by Marangoni surfers, i.e., self-propelled particles floating at an air-water interface. The spontaneous propulsion mechanism arising from these isotropic objects was first described qualitatively and, more recently, different numerical and analytical models were proposed [15,20,21]. On a qualitative level, particles act as chemical sources continuously releasing a surfactant substance in the surrounding water (in experiments this would typically be camphor), inducing surface tension gradients at the origin of Marangoni flows in their vicinity. Depending on the physical and geometrical parameters, a spontaneous symmetry breaking can occur so that a single particle would start to move at constant velocity in an unbounded space. Such a picture led to numerical models of camphor boats treating the swimmers as moving sources of camphor, which were successful in reproducing observations made in $1 \mathrm{D}$ confined systems [16,17,22,23].

The present study builds upon these works and proposes an implementation of multi-swimmer dynamics in a 2D and unconfined space so that the dynamics of the swimmers is fully unconstrained. Relaxing these constraints allows for the study of two-body interaction and reveals all the richness of the model when increasing the number of swimmers, for which all interactions are mediated by the camphor concentration field.

The article is structured as follows. In Sec. II, we introduce the model and show that the swimmers can be considered as tracers following the Marangoni flow filtered at their spatial scale. We then explain its numerical implementation, which combines the use of spectral methods to solve the transport 
equation for the camphor and Lagrangian interpolation using Gaussian filters to compute the velocity of the swimmers. We then show in Sec. III that the model reproduces the generic features of spontaneous swimming by symmetry breaking as observed in a point particle model [15] and characterize the interaction between two colliding swimmers in Sec. IV. We then address the multiparticle dynamics in Sec. V where we investigate the dispersion of the particles due to the random agitation generated by all the swimmers. In particular, we show that, although the present model is very simple, it reproduces the transition between a regime of dispersion by random agitation when the number of swimmers is moderate [7] to the regime of crystallization with imperfect hexagonal lattice for high swimmers density [12]. Finally, the last section is devoted to a discussion of the results and a conclusion.

\section{MODEL}

\section{A. Camphor field evolution}

The model we develop hereafter is inspired from symmetric surfers such as well-known floating camphor disks $[16,17,22,23]$. The main difference with previous numerical works is that we build here a $2 \mathrm{D}$ model so that the dynamics of the swimmers is less constrained than in one dimension, while being enough computationally efficient to study the multiparticle dynamics for very long timescales. To achieve this goal, we model the dynamics of the swimmers as an ensemble of floaters at location $\boldsymbol{R}_{i}(t)$, free to move on a surface $S$ while behaving as chemical sources of a scalar with surface concentration $\Gamma$ (called hereafter camphor concentration by analogy with experimental systems). In the presence of a surface flow $\boldsymbol{u}$, which may be a superposition of an external flow and the Marangoni flow caused by the sources, the scalar $\Gamma$ obeys an advection-diffusion equation in the presence of chemical reaction and sources

$$
\partial_{t} \Gamma+\nabla \cdot \boldsymbol{j}=-\alpha \Gamma+\sum_{i=1}^{N} s\left[\boldsymbol{r}-\boldsymbol{R}_{i}(t)\right] .
$$

In this equation $\boldsymbol{j}=-D_{0} \boldsymbol{\nabla} \Gamma+\boldsymbol{u} \Gamma$ is the total scalar current incorporating diffusive and advective contributions, $D_{0}$ being the camphor diffusivity, while $-\alpha \Gamma$ accounts for the sublimation of camphor at the surface [24] and $s(\boldsymbol{r})$ is the source term of camphor released by a single particle. Note that, except where otherwise mentioned, all variables, vectors, and operators are defined in the surface plane so that this equation may be regarded as averaged over the thickness of the water.

Experimentally, camphor is both advected by Marangoni flows and autodiffused with Péclet numbers of the order of $\mathcal{O}\left(10^{4}\right)$. Very long simulations of the system with realistic numbers would then be prohibitive, so that we chose to model the combination of both effects as a single effective diffusion process; this assumption is in accordance with experimental results [25-27], and reinforced by numerical and theoretical approaches [28,29]. We therefore set

$$
\boldsymbol{j}=-D \nabla \Gamma,
$$

where $D$ is an effective diffusion coefficient, orders of magnitude larger than the actual diffusion coefficient $D_{0}[28,29]$. We finally obtain

$$
\partial_{t} \Gamma=D \Delta \Gamma-\alpha \Gamma+\sum_{i=1}^{N} s\left(\boldsymbol{r}-\boldsymbol{R}_{i}\right) .
$$

\section{B. Marangoni flow}

In the absence of external driving, the flow in the liquid results from the interfacial forcing by surface tension gradients. These Marangoni flows thus relate to camphor distribution $\Gamma$. In the following, this relationship is taken as a linear form

$$
\boldsymbol{u}=-A \nabla \Gamma \text {. }
$$

Indeed, such a form naturally emerges from a lubrication approximation for the hydrodynamic problem. Within this framework and further, assuming a classical linear regime between the surface tension $\gamma$ and the concentration

$$
\gamma=\gamma_{0}-\beta \Gamma,
$$

we can show that the surface velocity $\boldsymbol{u}_{s} \equiv \boldsymbol{u}$ reads

$$
\boldsymbol{u}_{s}=-\frac{h \beta}{4 \eta} \nabla \Gamma
$$

where $\eta$ is the viscosity of water and $h$ the shallow water depth (see the Appendix for the detailed derivation).

\section{Swimmers' dynamics \\ 1. Camphor sources}

Swimmers of size $\sigma$ act as extended sources typically releasing camphor throughout their surface. We choose to model source terms as Gaussian functions centered on each swimmer

$$
s\left[\boldsymbol{r}-\boldsymbol{R}_{i}(t)\right]=\frac{s_{0}}{2 \pi \sigma^{2}} \exp \left(-\left[\boldsymbol{r}-\boldsymbol{R}_{i}(t)\right]^{2} / 2 \sigma^{2}\right),
$$

where $s_{0}$ is the average value of the source term. The use of such a smooth function is motivated by the numerical algorithm implemented to solve Eq. (3), which is based on spectral methods and uses the 2D fast Fourier transform with finite resolution. Indeed, using discontinuous functions such as gate functions as in [16,17,22,23], which used finite differences, would require truncation in spectral space, thus introducing spurious oscillations in all fields in the vicinity of the swimmers.

\section{Dynamics of swimmers}

In the presence of Marangoni flows (or surface tension gradients), a swimmer moving at velocity

$$
\boldsymbol{V}_{i}=\frac{d \boldsymbol{R}_{i}}{d t}
$$

will be submitted to the action of various forces such as capillary and viscous forces, respectively, denoted by $\boldsymbol{F}_{c}$ and $\boldsymbol{F}_{\eta}$. The capillary driving force writes

$$
\boldsymbol{F}_{c}=\oint_{\partial \mathcal{D}} \gamma \boldsymbol{n} d l=\iint_{\mathcal{D}} \nabla \gamma d S
$$

where $\partial \mathcal{D}$ is the perimeter of the swimmer and $\mathcal{D}$ its surface. In the case of a thin layer of fluid with depth $h$ and viscosity $\eta$, it is possible to express the capillary force acting on a 
swimmer covering a surface $\iint_{\mathcal{D}} d S$ (see the Appendix for more details). It reads

$$
\boldsymbol{F}_{c}=\frac{4 \eta}{h} \iint_{\mathcal{D}} \boldsymbol{u} \mathrm{d} S=\frac{4 \eta}{h}\langle\boldsymbol{u}\rangle_{\mathcal{D}}\left[\boldsymbol{R}_{i}(t), t\right] \iint_{\mathcal{D}} d S,
$$

which shows that the capillary force is proportional to the velocity of the swimmer averaged over its surface $\langle\boldsymbol{u}\rangle_{\mathcal{D}}\left(\boldsymbol{R}_{i}(t), t\right)$. In this lubrication regime, a similar result is obtained for the drag force

$$
\boldsymbol{F}_{\eta}=-\frac{4 \eta}{h} \boldsymbol{V}_{i} \iint_{\mathcal{D}} d S
$$

so that a single swimmer traveling at constant speed moves at the average velocity $\boldsymbol{V}_{i}=\langle\boldsymbol{u}\rangle_{\mathcal{D}}\left[\boldsymbol{R}_{i}(t), t\right]$.

One may wonder if the result derived above still holds when the system is composed of several swimmers so that the surface flow is no longer stationary in any reference frame. To this end one may turn to a more complete force balance such as the one derived by Maxey and Riley and Gatignol [30,31] for inertial particles. In the case of a material particle moving in three dimensions in a flow with velocity $\boldsymbol{u}$ and acceleration $\frac{D u}{D t}$, the Maxey Riley Gatignol equation takes the complex form

$$
\begin{aligned}
m_{p} \frac{d \boldsymbol{V}_{i}}{d t}= & m_{f}\left\langle\frac{D \boldsymbol{u}}{D t}\right\rangle+\frac{1}{2} m_{f}\left(\left\langle\frac{d \boldsymbol{u}}{d t}\right\rangle-\frac{d \boldsymbol{V}_{i}}{d t}\right) \\
& +\boldsymbol{F}_{c}+\boldsymbol{F}_{\eta}+\boldsymbol{F}_{\text {history }},
\end{aligned}
$$

where $m_{p}$ and $m_{f}$ are the mass of the particle and the mass of fluid corresponding to the volume of the particle. In this equation, the two first terms in the right-hand side (R.H.S.) are the acceleration of the fluid averaged over the particle and the added mass force, while the last corresponds to the history force which was proved to be negligible in turbulent flows [32]. In the case of a neutrally buoyant particle $\left(m_{p}=m_{f}\right)$, this equation has the property to have a trivial solution $V_{i}=$ $\langle\boldsymbol{u}\rangle$ so that such a particle has no inertia. This solution proved to be verified in practice in turbulent flows whatever the initial conditions [33]. This limiting case is specifically relevant for floaters because as they are less dense than the fluid, they displace a mass of fluid equal to their own mass so that $m_{f}=m_{p}$. Extrapolating this result established in three dimensions to our 2D case, swimmers should then be considered as a special case of neutrally buoyant particles. They have no inertia and follow the surface flow averaged over their surface so that their equation of motion reads

$$
\frac{d \boldsymbol{R}_{i}}{d t}=\langle\boldsymbol{u}\rangle_{\mathcal{D}}\left[\boldsymbol{R}_{i}(t), t\right] .
$$

Consistently with our modeling of the sources as Gaussian functions, we will define a finite-size particle of size $\sigma$ using the same Gaussian function. Such a particle will therefore have the velocity

$$
\frac{d \boldsymbol{R}_{i}}{d t}=\langle\boldsymbol{u}\rangle\left[\boldsymbol{R}_{i}(t), t\right]
$$

the average being obtained as a convolution $\langle\boldsymbol{u}\rangle(\boldsymbol{r}, t)=G * \boldsymbol{u}$ with the Gaussian Kernel

$$
G(\boldsymbol{r})=\frac{\exp \left(-\boldsymbol{r}^{2} / 2 \sigma^{2}\right)}{\iint_{S} \exp \left(-\boldsymbol{r}^{2} / 2 \sigma^{2}\right) d S},
$$

where $S$ is the surface over which we solve Eq. (3). Such modeling of finite-size particles using Gaussian filters is inspired from previous works $[34,35]$, which showed that results obtained with this model compares well with the experimental data of finite-size particles transported in turbulent flows [36-38].

The system of swimmers is then modeled by a closed set of three coupled equations for the camphor concentration $\Gamma$, the surface flow $\boldsymbol{u}$, and the swimmer positions $\boldsymbol{R}_{i}(t)$ :

$$
\begin{aligned}
& \partial_{t} \Gamma=D \Delta \Gamma-\alpha \Gamma+\frac{s_{0}}{2 \pi \sigma^{2}} \sum_{i=1}^{N} \exp \left[-\left(\boldsymbol{r}-\boldsymbol{R}_{i}\right)^{2} / 2\right], \\
& \boldsymbol{u}=-M \nabla \Gamma, \\
& \frac{d \boldsymbol{R}_{i}}{d t}=\langle\boldsymbol{u}\rangle\left(\boldsymbol{R}_{i}, t\right),
\end{aligned}
$$

where $D, \alpha, s_{0}, \sigma, M$, and $N$ are parameters which can be tuned independently. In this regime, the swimmers are entirely coupled through chemical interactions, ignoring hydrodynamic interactions.

\section{Dimensionless form \\ 1. Equations}

To reduce the number of free parameters in the simulation, we now introduce non-dimensional numbers. The typical lengthscale of the flow is $\sigma$, and the typical concentration heterogeneity has the size of a swimmer; therefore the typical time for such heterogeneity to get smeared out is

$$
t^{*}=\frac{\sigma^{2}}{D} \text {. }
$$

The heterogeneity is renewed constantly by the sources, so that, equating the first and last terms of Eq. (3) and using Eq. (7), we obtain a typical concentration $\Gamma^{*}$ as $\Gamma^{*} / t^{*} \sim$ $s_{0} / \sigma^{2}$, that is,

$$
\Gamma^{*} \sim \frac{s_{0}}{D}
$$

Note finally that the typical Marangoni velocity induced by camphor gradients can be obtained from Eq. (4) as $U_{M} \sim$ $A \Gamma^{*} / \sigma$.

We now introduce non-dimensional variables $\tilde{t}=t / t^{*}$, $\widetilde{\Gamma}=\Gamma / \Gamma^{*}, \widetilde{\boldsymbol{u}}=\boldsymbol{u} t^{*} / \sigma, \widetilde{\boldsymbol{r}}=\boldsymbol{r} / \sigma, \widetilde{\boldsymbol{R}}=\boldsymbol{R} / \sigma$ and operators $\widetilde{\nabla}=\sigma \nabla$ and $\widetilde{\Delta}=\sigma^{2} \Delta$, and we obtain the coupled equations

$$
\begin{aligned}
& \partial_{t} \widetilde{\Gamma}=\widetilde{\Delta} \widetilde{\Gamma}-\bar{\alpha} \widetilde{\Gamma}+\frac{1}{2 \pi} \sum_{i=1}^{N} \exp \left[-\left(\widetilde{\boldsymbol{r}}-\widetilde{\boldsymbol{R}}_{i}\right)^{2} / 2\right], \\
& \widetilde{\boldsymbol{u}}=-M \widetilde{\nabla} \widetilde{\Gamma}, \\
& \frac{d \widetilde{\boldsymbol{R}}_{i}}{d \widetilde{t}}=\langle\widetilde{\boldsymbol{u}}\rangle\left(\widetilde{\boldsymbol{R}}_{i}, \widetilde{t}\right) .
\end{aligned}
$$

with two dimensionless parameters

$$
\begin{aligned}
& M=\frac{A s_{0}}{D^{2}}=\frac{U_{M}}{\sigma} \frac{\sigma^{2}}{D}, \\
& \bar{\alpha}=\frac{\alpha \sigma^{2}}{D} .
\end{aligned}
$$

$M$ is the Marangoni number of the flow that compares the Marangoni-stress-induced flow transport to the diffusion 
transport; it thus appears as a Péclet number based on the typical Marangoni velocity $U_{M} \cdot \bar{\alpha}$ compares camphor evaporation rate to the camphor diffusion rate; it is essentially identical to a second Damköhler number for reactive systems.

\section{Numerical implementation}

The system (19) involves equations written both in the Eulerian framework for the concentration $\tilde{\Gamma}$, and in the Lagrangian framework for the position of the particles $\tilde{R}_{i}(t)$. The Eulerian equation for the concentration is solved with periodic boundary conditions using a pseudospectral method with an implicit treatment of the diffusion term. The grid size is $128^{2}$ for a square box of length $L=2 \pi$, with fixed diffusion $D=0.15$, camphor flux $s_{0}=1, \sigma=9.85 \times 10^{-2}$, variable attenuation $\alpha \in[0-10]$, and $A \in[0-10]$. Using such fine resolution ensures that the Marangoni flow filtered at the scale of the particles, $\langle\boldsymbol{u}\rangle=-A\langle\nabla \Gamma\rangle$, which is computed in spectral space by multiplication with the Fourier transform of the normalized Gaussian function defined in Eq. (15) is resolved with high accuracy. Lagrangian velocities of particles can then be obtained by spline interpolation of $\langle\boldsymbol{u}\rangle$ at particle positions in physical space with a relative error smaller than $10^{-3}$. Both Eulerian and Lagrangian equations use an identical Adams-Bashford temporal scheme of order 2 with a timestep $d t=5 \times 10^{-3}$ so that non-dimensional parameters are $\widetilde{L}=63.8, \widetilde{\alpha} \in[0-0.65], M \in[0-450]$, and $\widetilde{\mathrm{dt}}=$ $7.7 \times 10^{-2}$. The code, implemented in MATLAB as a modified version of [39], can run on common desktop computers so as to obtain very long trajectories in the statistically stationary regime when using $\mathcal{O}(50)$ active particles.

\section{MARANGONI SWIMMING: SINGLE SWIMMER}

A single swimmer is introduced in the box, with an initial camphor field $\widetilde{\Gamma}=0$ and a vanishingly small velocity $\widetilde{V}_{0}=$ $10^{-3}$ in the $\bar{y}$ direction. We first set $\bar{\alpha}=0.13$, a regime in which the effect of camphor sublimation is weak compared to camphor diffusion. The Marangoni number is $M \in[0,480]$.

As evidenced in Fig. 1(a), a swimming transition is observed above a critical Marangoni number $M_{c} \approx 23.9$. Below $M_{c}$ the final steady state is motionless with a camphor concentration remaining symmetric (see the inset, left with $M=23.1<M_{c}$ ). This concentration generates axisymmetric Marangoni flows as can be observed experimentally with a fixed source of surfactant [40], but does not yield selfpropulsion because the concentration gradient is exactly zero at the location of the swimmer. Above $M_{c}$, a stationary state without motion is unstable. Given the very small initial velocity, a symmetry breaking occurs (as in the experiments) so that the surfer velocity increases rapidly and reaches a constant value $\widetilde{V}_{s}$. This symmetry breaking can be traced back to the camphor concentration field displayed as middle and right insets in Fig. 1(a) in the case of a swimmer moving toward the top. After a transient during which the camphor cloud grows and looses its symmetry, a steady-state distribution is reached in the reference frame of the surfer which is distorted towards its rear, all the more that $M$ increases.

More into details, Figs. 1(a) and 1(b) show the swimming velocity evolution with $M$. As can be seen, the bifurcation
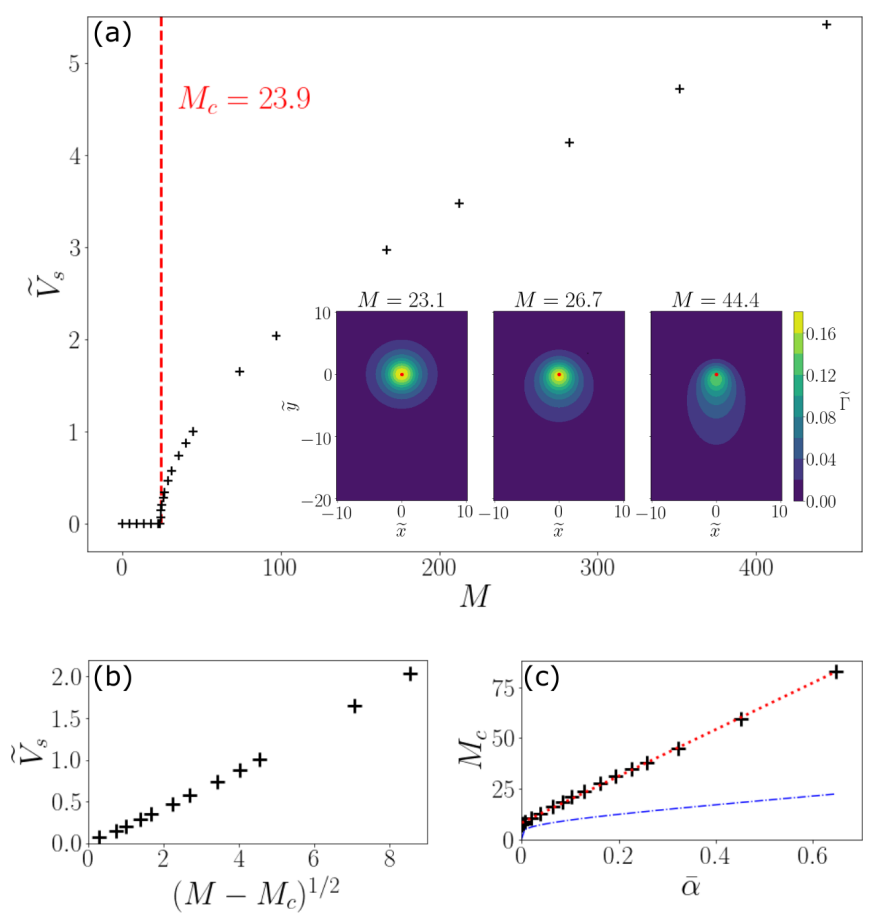

FIG. 1. Spontaneous swimming of single surfers. (a) Velocity $\widetilde{V}_{s}$ for different $M$, keeping $\bar{\alpha}=0.13$ : swimming is observed above a critical value $M_{c}=23$.9. Inset: Isoconcentration lines of the camphor field for different $M$ just below $(M=23.1$, no swim) just above ( $M=26.7)$ and far above $(M=44.4)$ the swimming threshold. The center of the swimmer is indicated by a red circle, its swimming direction is towards the top. (b) $\widetilde{V}_{s}$ evolution in the vicinity of the swimming threshold showing a $\left(M-M_{c}\right)^{1 / 2}$ dependency. (c) Critical Marangoni number $M_{c}$ as a function of $\bar{\alpha}$ : red dotted line shows an affine regression; blue dash-dotted line is the toy-model prediction $M_{c}=4 \pi / K_{0}(\sqrt{\bar{\alpha}})[15]$.

occurs through a supercritical pitchfork transition, with $\widetilde{V}_{s}$ scaling as $\left(M-M_{c}\right)^{1 / 2}$ above the threshold. In addition the influence of $\bar{\alpha}$ on the critical Marangoni number $M_{c}$ is shown in Fig. 1(c): the stronger the evaporation (large $\bar{\alpha}$ ) the higher $M_{c}$. Indeed, a stronger evaporation rate decreases the extent of the camphor field at the steady state. For the advective transport to dominate diffusion over the cloud extent and to be able to trigger a symmetry breaking thus requires more intense Marangoni effects and hence larger $M$ values.

Overall, these different behaviors are consistent with analytical predictions that can be drawn from an even simpler toy (a model where the surfer is modeled as a camphor pointsource) [15]. Within this approach, a swimming transition in the form of a supercritical pitchfork bifurcation is obtained with $M_{c}=4 \pi / K_{0}(\sqrt{\bar{\alpha}})$ with $K_{0}$ the modified Bessel function of the second kind. While the qualitative picture, together with the correct orders of magnitudes, are successfully captured by this simplified framework, Fig. 1(c) shows that the present model predicts a higher swimming threshold whatever $\bar{\alpha}$. This is a consequence of the spatial extension of our source, which induces that spontaneous symmetry breaking is harder to obtain than with a point source. 

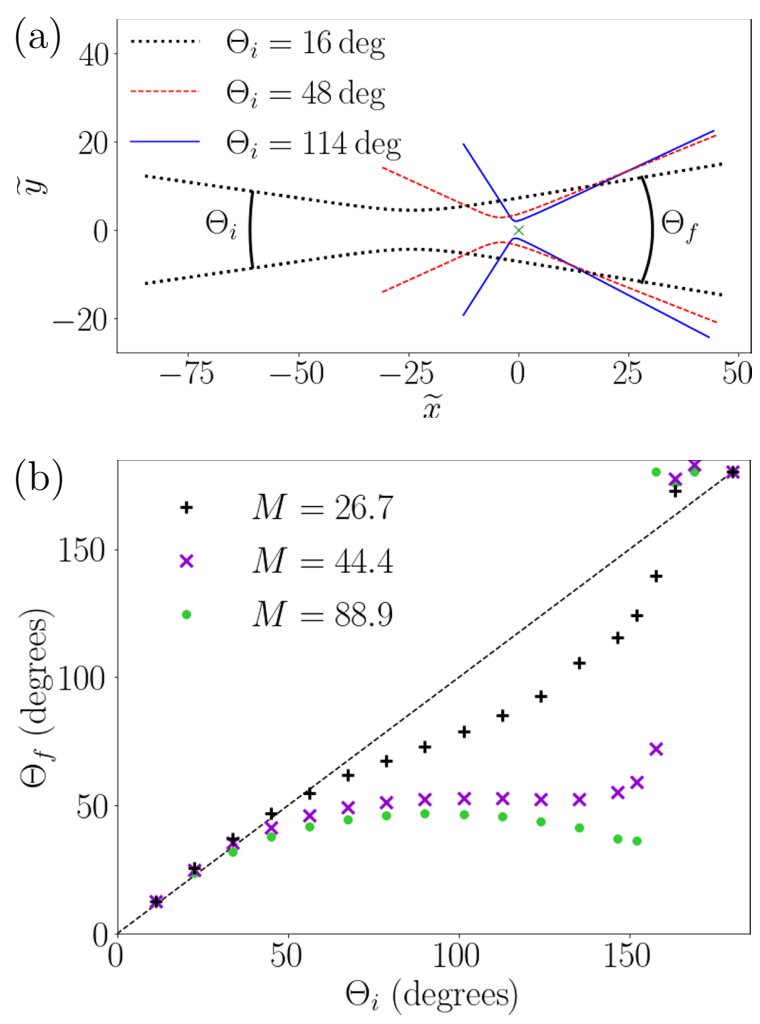

FIG. 2. (a) Trajectories of swimmers planned to collide synchronously at the origin for different initial angle $\Theta_{i} \in\{16,48,114\}$. $M=44.4, \bar{\alpha}=0.13$. (b) Angle $\Theta_{f}$ after the synchronous collision with respect to the angle $\Theta_{i}$ for $M \in\{6.7,11.1,22.2\}$ with $\bar{\alpha}=0.13$.

\section{TWO-BODY INTERACTION}

With the swimming properties of single surfers defined, the next step is to address the two-body interactions. This is readily done according to Eq. (19) by introducing two sources in the periodic box. Box size and surfer initial separation are taken large enough so that they initially behave as isolated swimmers.

To accumulate systematic information and control the interaction parameters, we first consider configurations with vanishing impact parameter: the surfers are initially located at equal distance from the center; their velocities are identical in magnitude, equal to that of a single swimmer, and both point towards the box center. Surfers trajectories corresponding to swimming characteristics $M=44.4$ and $\bar{\alpha}=0.13$ are shown in Fig. 2(a) for different initial angle of collision $\Theta_{i}$. While the collision from nearly parallel surfers look quasispecular, the behavior evolves at higher angles with exiting trajectory that seems to hardly depend on the initial $\Theta_{i}$.

These results are summarized in Fig. 2(b), showing the final exit angle $\Theta_{f}$ as a function of the initial collision angle $\Theta_{i}$, for different swimming parameter $M$, and fixed $\bar{\alpha}=0.13$. Three regimes can be identified while increasing the collision angle. As already mentioned, for weak angles $\Theta_{i}<50^{\circ}$, the collision is almost specular with surfers exchanging their velocity directions. This regime is followed by a wide range of collision angles $\Theta_{i} \in\left[50^{\circ}, 150^{\circ}\right]$ where the final separation angle is almost constant around $\Theta_{f} \simeq 50^{\circ}$, therefore corresponding to a partial alignment regime. Note that, unlike alignment mechanisms in other active systems with an intrinsic asymmetry [41], here the alignment seems bounded: it kicks in only at high-enough angle, and aligns to within a minimum angle. Note also that this partial alignment tends to disappear for smaller values of $M-M_{c}$ where the chemical cloud asymmetry is weaker; in this case the collision remains closer to specular throughout the first two regimes. Finally, near head-on collisions with $\Theta_{i}>150^{\circ}$ result in an antialignment with a final trajectories showing opposite directions with $\Theta_{f} \simeq 180^{\circ}$.

As already mentioned, model investigations to date with Marangoni surfers have essentially dealt with 1D approaches of often asymmetric particles; consequently, the state-of-the-art for interactions also considers 1D systems $[25,42,43]$. Recent advances have, however, been obtained for a closely connected case: active droplets embedded in a three-dimensional (3D) liquid phase that also exhibit swimming by spontaneous symmetry breaking. In this system, which proved more amenable to analytical development of the full problem than surfers, the properties of 2D binary interactions were recently addressed [44-46]. The collision data reported for these active droplets are in agreement with experimental investigations [47,48], and display properties very similar to those presented here. This suggests that the interaction of symmetric active swimmers share generic features that make them distinct from the mostly described active systems [10,49].

Finally, let us mention that fully generic interaction events also include the case of finite impact parameters. As compared to the previous case, this can be viewed as launching the two surfers of initial velocity angle $\Theta_{i}$, at different times. With increasing delay, the interaction progressively occurs through the chemical wake until the delay is large enough so that the surfers move independently. This generic situation was fully explored for active droplets: while for small delays the collision features are little changed, they overall exhibit a rich set of rebounds or crossing behaviors [46]. As for the symmetric collisions, the present system displays qualitatively the same features (not shown here).

\section{COLLECTIVE BEHAVIORS}

We now examine the collective properties of our Marangoni surfer model. While intensively studied for asymmetric swimmers, little investigations have addressed the dynamics and collective response of symmetric active particles. A few experimental articles dealt with the dynamics of semi-dilute to concentrated assemblies of interfacial surfers, either in one dimension [50] or in two dimensions [7,12,51]. Theoretically, the collective case of chemical sources at a fluid interface has been considered, but without allowing for their self-propulsion [52-54]. In addition, very recent studies investigate the $2 \mathrm{D}$ dynamics of semi-dilute active droplets showing caging effects [48]. In addition, confinement-dependent clustering and polar ordering were reported in a few experimental and numerical approaches of these droplet systems [55-57]. 


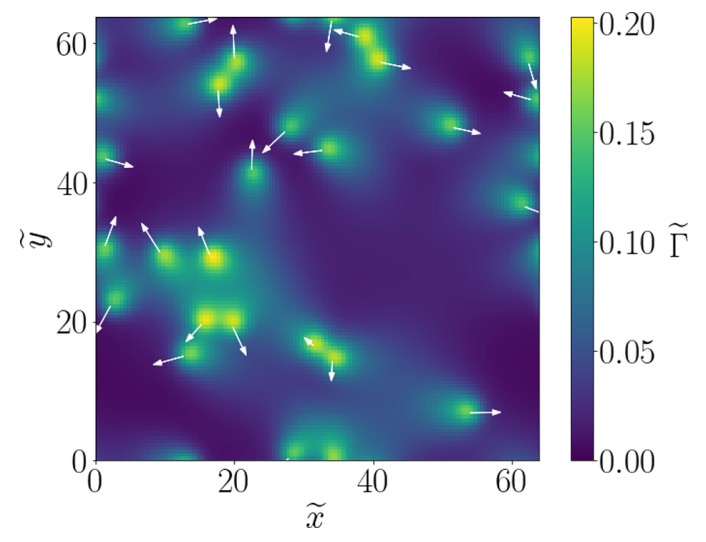

FIG. 3. Instantaneous camphor concentration field obtained for $N=25$ swimmers, $M=44.4$, and $\bar{\alpha}=0.13$, long enough after initialization to avoid any memory from the initial state. Each white arrow starts at the center of a swimmer and its direction and length indicates its velocity.

\section{A. Transport properties}

\section{Characterization}

In the following, we consider $N$ identical surfers with characteristics set to $M=44.4$ and $\bar{\alpha}=0.13$. We define their surface density in the simulation box as $[7,40]$

$$
\Phi=\frac{N \pi \sigma^{2}}{L^{2}}
$$

Varying $N$ from 5 to 200 corresponds to densities $\Phi$ from 1.9 to $76 \%$. Figure 3 presents a snapshot of the surface concentration field obtained for $N=25(\Phi \simeq 9.5 \%)$, with the origin of each white arrow being the surfer center and the length its instantaneous velocity.

The surfers collective dispersion properties are characterized via the mean square displacement (MSD) as a function of time lag $\Delta \widetilde{t}$

$$
\sigma_{\text {msd }}^{2}(\Delta \widetilde{t})=\frac{1}{N} \sum_{i=1}^{N}\left\langle\left(\widetilde{\boldsymbol{R}}_{i}\left(\widetilde{t}_{0}+\Delta \widetilde{t}\right)-\widetilde{\boldsymbol{R}}_{i}\left(\widetilde{t}_{0}\right)\right)^{2}\right\rangle_{\tau_{0}},
$$

where $\langle\cdot\rangle_{\tau_{0}}$ is an average over every possible $\widetilde{t}_{0}$ values. The computed MSDs are shown in Fig. 4(a) for different numbers of swimmers $N$. Overall, the dynamics in the multisurfers system resembles the classical one of single active Brownian particles [58,59]: a short-term ballistic regime $\left(\sigma_{\text {msd }}^{2} \propto \Delta \widetilde{t}^{2}\right)$ followed by a long-term effective diffusion regime $\left(\sigma_{\mathrm{msd}}^{2} \propto\right.$ $\Delta \widetilde{t})$. However, the ballistic dynamics of the single surfer shows that there is no rotational diffusion of the swimming direction. Randomization of the orientation occurs only through surfers interactions, and indeed occurs earlier (shorter $\Delta \widetilde{t}$ ) at higher densities, in accordance with experimental observations on camphor disks [7].

The ballistic velocities $\widetilde{v}^{\prime}$ and effective diffusivities $\widetilde{D}_{\text {eff }}$ are obtained from fits of the associated asymptotic regimes for each $N$ according to

$$
\sigma_{\mathrm{msd}}^{2} \approx \widetilde{v}^{\prime 2} \Delta \widetilde{t}^{2} \quad \text { at small } \Delta \widetilde{t}
$$

and

$$
\sigma_{\mathrm{msd}}^{2} \approx 4 \widetilde{D}_{\mathrm{eff}} \Delta \widetilde{t} \quad \text { at large } \Delta \widetilde{t},
$$
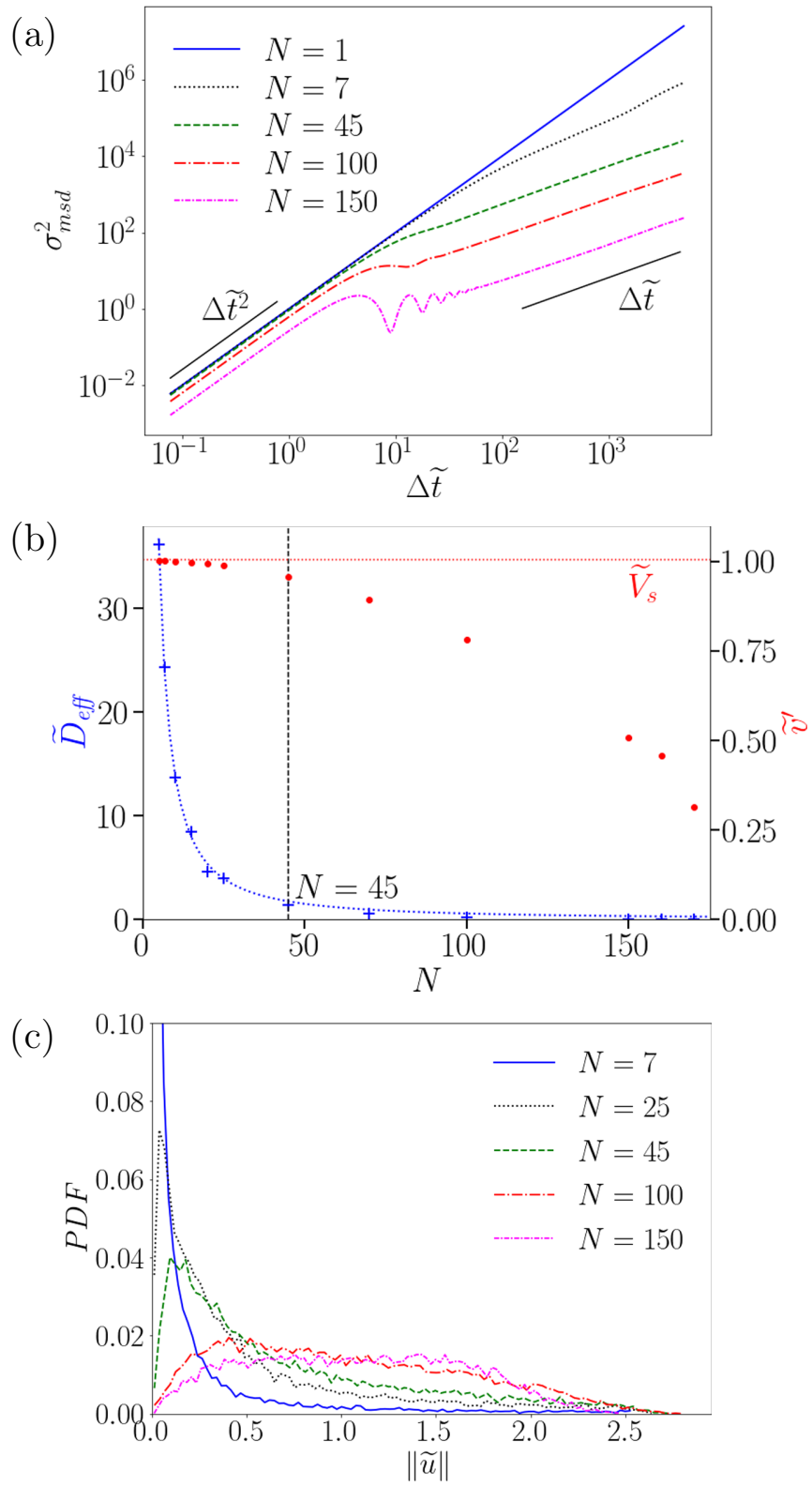

FIG. 4. (a) Mean square displacement of the swimmers for various number of particles in the box. $M=44.4, \bar{\alpha}=0.13$. (b) Variation of $\widetilde{D}_{\text {eff }}$ (blue marks) and $\widetilde{v}^{\prime}$ (red dots), the characteristic coefficients of respectively the diffusive and ballistic regime with respect to the number of swimmers $N$. The dotted horizontal line indicates the velocity of a single swimmer $\widetilde{V}_{s}$; the dotted blue line shows a power-law fit $\widetilde{D}_{\text {eff }}=34 N^{-1.55}$. (c) Probability density function of velocity field magnitude for different number of swimmers.

respectively. The variation of both coefficients is displayed in Fig. 4(b) as a function of the number of swimmers $N$. For low surface density $(N \leqslant 45 ; \Phi \leqslant 17 \%)$, the ballistic velocity $\widetilde{v}^{\prime}$ remains unchanged, equal to that of a single surfer. In this regime, swimmers behave as if isolated except for interaction events which modify their direction. At higher densities, the ballistic regime eventually becomes density dependent: the velocity decreases with increasing crowding.

This transition between low and high density regimes can be simply understood on qualitative grounds: one may 
expect that permanent interaction between surfers occurs when chemical wakes fill the whole surface. Indeed, Fig. 3 shows a snapshot of the concentration field at $N=25$, before the transition, with significant remaining zones with no chemical pollution. This translates in the probability distribution function (PDF) of the surface velocity amplitude [Fig. 4(c)] by a finite probability to find locations with no Marangoni flow. When reaching the transition around $N=45$ this feature disappears and no remaining fraction of the surface is motionless.

In the high density regime, one may be tempted to associate the slowing down of ballistic motion to a smoothing of chemical gradients. However, this is not what comes out of the previous PDFs in Fig. 4(c), which show that the probability of high surface velocities increases with $N$. Indeed, it is really the hindering of the motion by surrounding neighbors that is responsible of the dynamics slowing down. This cage effect characteristic of dense systems shows up in the high density regime [Fig. 4(a), $N>100$ ] as a short plateau between the ballistic and diffusive regimes [60]. This is in line with recent experimental reports on active drops dynamics in semi-dilute regimes [48]. Interestingly, this plateau is accompanied in the present system by oscillations in the MSD, arising from a rattling motion of the caged particles.

As already mentioned, in all $N>1$ configurations, surfers interactions induce a randomisation of the swimming orientation at large time which results in an effective diffusion regime in the MSD. Classically, we define the decorrelation time $\tilde{\tau}_{R}$ as the cross-over time between ballistic and diffusive regimes. From Eqs. (23) and (24), we obtain the relation

$$
\widetilde{D}_{\text {eff }} \sim \widetilde{v}^{\prime 2} \widetilde{\tau}_{R} / 4
$$

Intuitively, we expect the time $\widetilde{\tau}_{R}$ to decrease with $N$ due to the higher rate of collisions, in agreement with results in Fig. 4(a). With both the velocity $\widetilde{v}^{\prime}$ and the time $\widetilde{\tau}_{R}$ decreasing function of $N$, so is the effective diffusivity. A fit of the numerical data shows that this decay is consistent with a power-law behavior $\widetilde{D}_{\text {eff }} \propto N^{-1.55}$.

\section{Persistent random walk model}

Up to moderate densities, surfers essentially propel at the velocity of isolated particles and have their swimming direction randomized by successive collision events. It is therefore natural to compare this dynamics to a persistent random walk model. Because of the nature of reorientations, two elements need be specified for the random walk (i) the frequency $1 / \widetilde{\tau}_{R}$ of collision events and (ii) the statistics of the direction changes upon collision. The collision frequency can be estimated based on classical gas kinetics arguments, and corresponds to the rate at which a swimmer sweeps an area containing on average one other surfer

$$
\left(\frac{N}{\widetilde{L}^{2}}\right) \tilde{\ell}_{\perp} \widetilde{v}^{\prime} \widetilde{\tau}_{R}=1,
$$

where $\tilde{\ell}_{\perp}$ stands for the collision cross-section. In the following, it is taken as the transverse extension of the chemical wake associated to the surfers. Choosing a threshold at $5 \%$ of the maximum concentration sets $\widetilde{\ell}_{\perp}=13$ for the parameters considered $(M=44.4, \bar{\alpha}=0.13)$, see also Fig. 3. For the statistics of reorientations, one assumes that all collisions
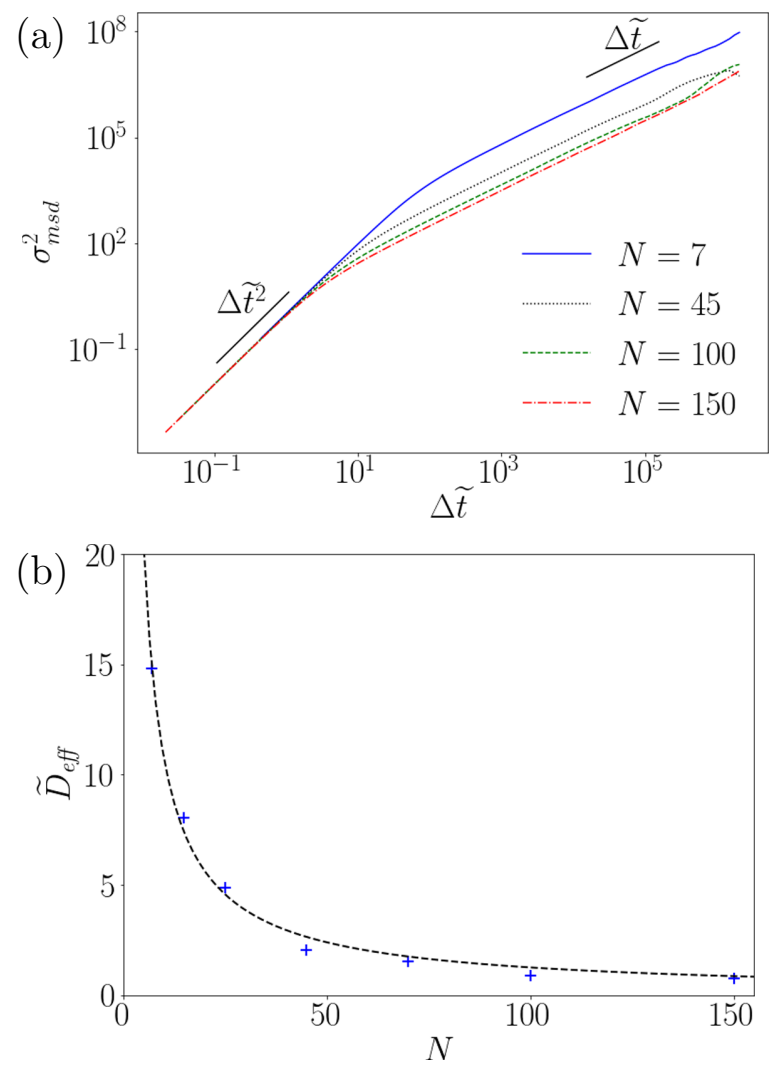

FIG. 5. Persistent random walk model. (a) Mean-square displacement for different numbers of swimmers $N$. (b) Variation of $\widetilde{D}_{\text {eff }}$ with $N$. Dotted black line indicates a power-law fit $\widetilde{D}_{\text {eff }}=93 N^{-0.94}$.

are symmetric so that for a given initial angle $\Theta_{i}$ drawn in a uniform distribution in $\left[0^{\circ}, 360^{\circ}\right]$, the deviation angle $\Theta_{f}$ follows according to Fig. 2(b).

Figure 5(a) shows MSDs computed according to this simple random walk model, for different number $N$ of surfers. As expected from persistent random walk, MSDs exhibit a ballistic and diffusive regime, with effective diffusivity decreasing with $N$ (Fig. 5).

By construction, the present approach can only be compared to the moderate density regime described previously: $\widetilde{v}^{\prime}$ is independent of $N$ and only binary collisions are considered, forbidding any caging regime. Overall this simple approach yields consistent magnitude for the effective diffusivities in the relevant range $N \lessgtr 45$ (Figs. 4 and 5), although the power-law decrease of $\widetilde{D}_{\text {eff }}$ is weaker (exponent -0.94 versus $-1.55)$. In the present approach, the $N$ dependency is entirely encoded in the mean free path argument for binary collisions. Potential origins of this discrepancy are the possibility of many (three) body interactions, or the existence of asymmetric collisions, not captured in this model.

\section{B. Active phase transition}

Looking in more detail at the transition between the ballistic and diffusive regimes in Fig. 4(b), we observe for $N=150$ some oscillations. Indeed, for this parameter the particles are almost stuck between their neighbors [Fig. 6(a)], 

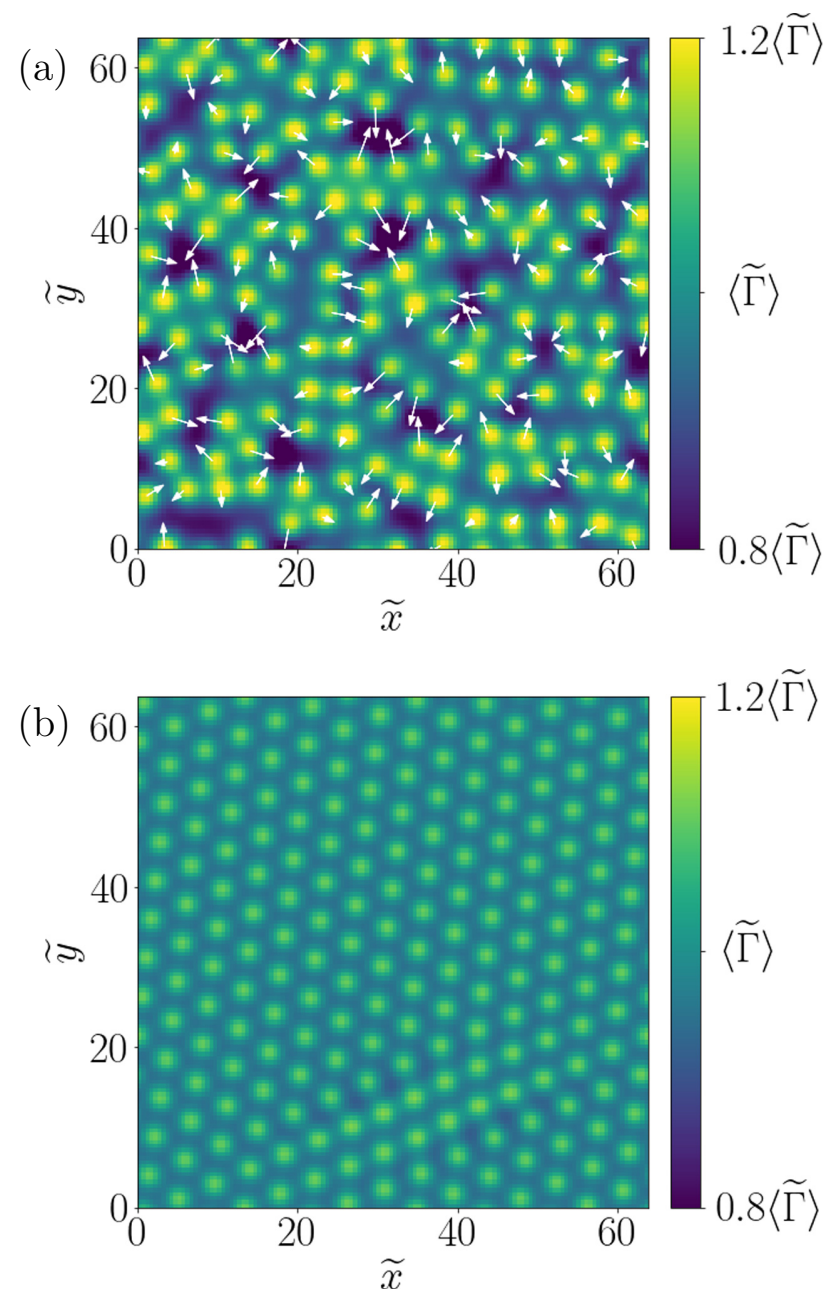

FIG. 6. Camphor concentration field obtained for $M=44.4$ and $\bar{\alpha}=0.13$. (a) $N=150$; white arrows indicate the velocity of the swimmers. (b) $N=200$; the system is almost crystallized.

and oscillate in this trap with a typical amplitude equal to their diameter, diffusing only over very long timescales.

Finally, when increasing up to $N=200(\Phi=76 \%)$, swimmers slowly self-organize to occupy the available space, being regularly spaced and almost motionless despite the periodic boundary conditions [see Fig. 6(b)]. Because the system clearly evolves toward a hexagonal lattice, we define an orientational hexagonal order parameter for each swimmer $j$, denoted by $\Psi_{6, j}$, as in $[61,62]$ :

$$
\Psi_{6, j}=\frac{1}{6} \sum_{k \in n_{j}} \exp \left(6 i \Theta_{j, k}\right)
$$

where $n_{j}$ is the set of six nearest neighbors of the particle $j$ and $\Theta_{j, k}$ is the angle of the vector between particle $j$ and particle $k$ with respect to the reference frame. Averaging over $N_{j}=2000$ swimmers (taken at different times), we obtain the average hexagonal order parameter $\Psi_{6}$ :

$$
\Psi_{6}=\frac{1}{N_{j}}\left|\sum_{j=1}^{N_{j}} \Psi_{6, j}\right|
$$
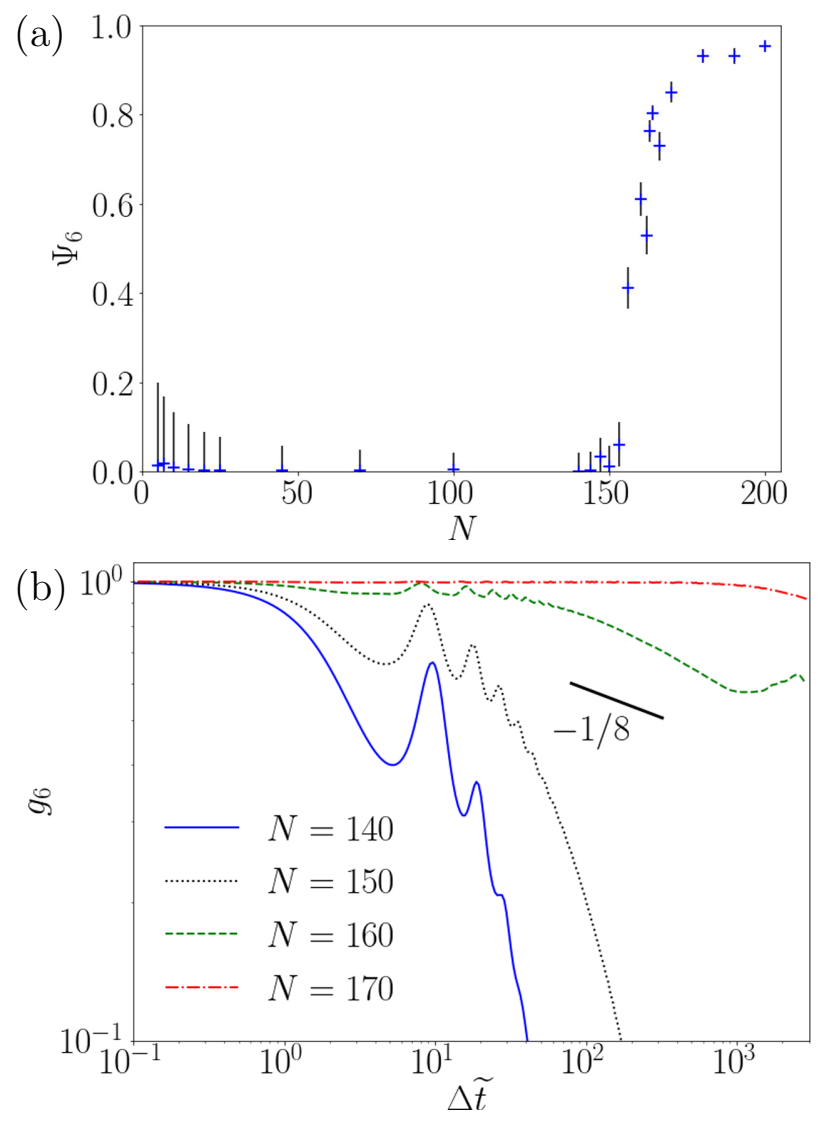

FIG. 7. (a) Mean hexagonal order parameter with respect to the number of swimmers. We observe a phase transition between no order $(N<150)$ and an almost crystallized state $(N=200)$. (b) Evolution of $g_{6}$ with respect to the time for different numbers of swimmers. The $t^{-1 / 8}$ behavior recalls the expected trend for the case of a colloidal hexatic phase.

Figure 7(a) shows the variations of $\Psi_{6}$ as a function of the number of swimmers $N$ in the periodic box: we clearly see that an hexagonal organization appears around $N=150$, indicating a transition between two phases. This transition is analogous to the liquid-hexatic transition observed for thermal passive particles and is already well described [62-64]. The same transition was also described for active Brownian particles [65], yet we report it here for an athermal system. To characterize better the transition, we use $g_{6}$, the autocorrelation function of $\Psi_{6}$, defined as

$$
g_{6}(\Delta t)=\operatorname{Re}\left(\frac{\left\langle\Psi_{6, j}\left(t_{0}+\Delta t\right) \cdot \Psi_{6, j}^{*}\left(t_{0}\right)\right\rangle_{t_{0}, j}}{\left\langle\left|\Psi_{6, j}\right|^{2}\right\rangle_{t_{0}, j}}\right) .
$$

It is now well known [63] that this function, at large timescales, exhibits in the colloidal hexatic phase a power law $g_{6} \propto \Delta \tilde{t}^{-\eta_{6} / 2}$ where $\eta_{6}$ depends on the density of particles and is equal to $1 / 4$ at the transition. Figure 7(b) shows on a logarithmic scale the evolution of $g_{6}$ for different number of swimmers $N$; as for colloidal phases we observe for this athermal system a clear evolution of $g_{6}$ with $N$. Understanding how the transition is influenced by the activity parameters $(M, \bar{\alpha})$, investigating the possibility of a full mapping to the 
at-equilibrium hexatic transition, are open questions that we hope will generate future theoretical investigations.

\section{SUMMARY AND CONCLUSION}

In the present article, we proposed a $2 \mathrm{D}$ model of Marangoni surfers in unconfined space. The model is based on the use of an effective diffusion equation for the transport of camphor on the surface, with a sublimation term and Gaussian source terms representing the swimmers which in turn follow the Marangoni flow filtered at their spatial scale. The present model is fully unconstrained so that several interesting problems can be addressed with the same code.

In the most simple case of a single swimmer we demonstrated that this model reproduces the swimming instability which arises following a supercritical bifurcation similar to the one obtained in several models from the literature $[15,17,22]$. We then showed that it allows to reproduce most of the known features of the multiparticle case, from the regime of dispersion enhancement by random agitation when the number of swimmers is moderate [7] to the regime of crystallization with imperfect hexagonal lattice when the swimmers reach high densities [12]. The imperfection is due to the geometry of the computational domain, which imposes a periodicity along $x$ and $y$ axes. It could be possible to obtain an ideal crystal by using a hexagonal computational domain which could be achieved at low cost with penalization methods [66].

Finally, the main disagreement with the experimental observations reported in [7] is the absence in the simulation of a turbulent signature in the swimmers velocities. Such effect may be due to the strong assumptions made to get a closed model, for which one only needs to solve the transport equation for the camphor without solving the Navier-Stokes equation to obtain the velocity. Indeed, in the lubrication regime where the Reynolds number is vanishingly small, the Marangoni flow is purely potential as it is proportional to the gradient of camphor concentration. To get a more realistic surface flow, one may relax the lubrication approximation to obtain a weakly nonlinear model of the 3D flow in the subphase, which would then have both potential and rotational contributions. In this regime, the system would still be closed for the camphor concentration so that it would be possible to simulate for a very long time even in the multiparticle regime characterized by strong spatiotemporal fluctuations.

\section{ACKNOWLEDGMENTS}

This work was supported by the French research programs ANR-16-CE30-0028 and IDEXLYON of the University of Lyon in the framework of the French program "Programme Investissements d'Avenir" (ANR-16-IDEX-0005).

\section{APPENDIX: RELATION BETWEEN MARANGONI FLOW AND CAMPHOR CONCENTRATION}

We hereafter show how the simple relationship between camphor concentration and Marangoni-driven surface flow [Eq. (4)] adopted in the model, naturally arises in the limit of lubrication flows. Unlike previous calculations, we here con- sider a 3D problem, with camphor (bulk) concentration $c$ in the subphase of thickness $h \ll \sigma$. Assuming a Henry isotherm for dilute camphor regimes, the surface tension writes

$$
\gamma=\gamma_{0}-\varepsilon c(x, y, z=h),
$$

where $\varepsilon$ is a constant. This leads to

$$
\nabla_{\|} \gamma=-\left.\varepsilon \nabla_{\|} c\right|_{(z=h)},
$$

where $\nabla_{\|}$is the gradient operator components parallel to the interface.

To proceed toward a simple closed form for the induced surface velocity, we consider as already mentioned, the limit of the shallow waters. In this limit camphor diffusion across the liquid depth is very fast compared to the lateral transport so that

$$
c(x, y, z) \approx c(x, y)
$$

from which we can define a surface concentration as

$$
\Gamma=h c(x, y) \text {. }
$$

For the flow field, in the lubrication limit, vertical velocity is negligible compared to horizontal ones

$$
\boldsymbol{u} \approx u_{x} \boldsymbol{e}_{\boldsymbol{x}}+u_{y} \boldsymbol{e}_{\boldsymbol{y}}=\boldsymbol{u}_{\|}
$$

Accordingly the pressure gradient along the liquid depth is ignored so that the Stokes equation reduces to

$$
\eta \frac{\partial^{2} \boldsymbol{u}_{\|}}{\partial z^{2}} \approx \nabla_{\|} P(x, y)
$$

which integrates to [67]

$$
u_{\|}(x, y, z)=\left(\frac{z^{2}-z h}{2 \eta}\right) \nabla_{\|} P+\frac{z}{h} \boldsymbol{u}_{s},
$$

with $\eta$ the fluid dynamic velocity, and $\boldsymbol{u}_{\boldsymbol{s}} \equiv \boldsymbol{u}(x, y, z=h)$ the velocity field at the interface. Neglecting surface deformation so that $h$ is constant over $(x, y)$, we define the depth-average velocity field $\bar{u}$ as

$$
\overline{\boldsymbol{u}}(x, y)=\frac{1}{h} \int_{0}^{h} u_{\|}(x, y, z) d z .
$$

Using the divergence-free condition for $\boldsymbol{u}$ together with the vanishing of the vertical velocity at $z=0$ and $h$, we obtain

$$
\nabla_{\|} \cdot \bar{u}=0
$$

which in combination with Eq. (A7) leads to

$$
\Delta_{\|} P=\frac{6 \eta}{h^{2}} \nabla_{\|} \cdot \boldsymbol{u}_{s} .
$$

The Marangoni boundary condition at the interface leads to

$$
\nabla_{\|} \gamma=\left.\eta \frac{\partial \boldsymbol{u}_{\|}}{\partial z}\right|_{(z=h)},
$$

and using Eq. (A7):

$$
\nabla_{\|} \gamma=-\frac{h}{2} \nabla_{\|} P+\eta \frac{u_{s}}{h} .
$$

The curl of this equation gives $\nabla \times \boldsymbol{u}_{s}=0$. Consequently, there exists a function $\phi$ such that $\boldsymbol{u}_{s}=\nabla_{\|} \phi$. Injecting this 
last relation into Eq. (A10) leads to

$$
\Delta_{\|} P=\frac{6 \eta}{h^{2}} \Delta_{\|} \phi
$$

We then deduce

$$
P=\frac{6 \eta}{h^{2}} \phi+\psi
$$

where $\psi$ is solution of $\Delta_{\|} \psi=0$. Assuming that $\psi=$ $f(r) \cos (\theta)$, possible solutions are $f(r) \propto 1 / r$ or $f(r) \propto r$. The last one can be discarded due to relaxation of the velocity field far from the disk. To avoid an infinite value of the field in $r=0$, the only solution left is $\psi=0$. Finally, Eq. (A12) reduces to

$$
\nabla_{\|} \gamma=4 \eta \frac{\boldsymbol{u}_{s}}{h}
$$

Combining equations (A2), (A4), and (A15), we eventually obtain the relation

$$
\boldsymbol{u}_{s}=-\frac{\varepsilon}{4 \eta} \nabla_{\|} \Gamma \text {. }
$$

Because the surface concentration reads $\Gamma=h c$, we have the relation $\varepsilon \equiv h \beta$ for the surface tension dependency with bulk and surface concentration. Finally, we indeed obtain the relationship chosen in our bi-dimensional model Eq. (4) providing $A=h \beta /(4 \eta)$.
[1] C. Dombrowski, L. Cisneros, S. Chatkaew, R. E. Goldstein, and J. O. Kessler, Self-Concentration and Large-Scale Coherence in Bacterial Dynamics, Phys. Rev. Lett. 93, 098103 (2004).

[2] H. H. Wensink, J. Dunkel, S. Heidenreich, K. Drescher, R. E. Goldstein, H. Löwen, and J. M. Yeomans, Meso-scale turbulence in living fluids, Proc. Natl. Acad. Sci. USA 109, 14308 (2012).

[3] M. C. Marchetti, J. F. Joanny, S. Ramaswamy, T. B. Liverpool, J. Prost, M. Rao, and R. A. Simha, Hydrodynamics of soft active matter, Rev. Mod. Phys. 85, 1143 (2013).

[4] A. Doostmohammadi, T. N. Shendruk, K. Thijssen, and J. M. Yeomans, Onset of meso-scale turbulence in living fluids, Nat. Commun. 8, 15326 (2016).

[5] P. Guillamat, J. Ignés-Mullol, and F. Sagués, Taming active turbulence with patterned soft interfaces, Nat. Commun. 8, 564 (2017).

[6] K.-T. Wu, J. B. Hishamunda, D. T. N. Chen, S. J. DeCamp, Y.-W. Chang, A. Fernández-Nieves, S. Fraden, and Z. Dogic, Transition from turbulent to coherent flows in confined threedimensional active fluids, Science 355, eaal1979 (2017).

[7] M. Bourgoin, R. Kervil, C. Cottin-Bizonne, F. Raynal, R. Volk, and C. Ybert, Kolmogorovian Active Turbulence of a Sparse Assembly of Interacting Marangoni Surfers, Phys. Rev. X 10, 021065 (2020).

[8] C. Bechinger, R. D. Leonardo, H. Löwen, C. Reichhardt, G. Volpe, and G. Volpe, Active particles in complex and crowded environments, Rev. Mod. Phys. 88, 045006 (2016).

[9] É. Fodor and M. C. Marchetti, The statistical physics of active matter: From self-catalytic colloids to living cells, Physica A 504, 106 (2018).

[10] T. Vicsek, A. Czirók, E. Ben-Jacob, I. Cohen, and O. Shochet, Novel Type of Phase Transition in a System of Self-Driven Particles, Phys. Rev. Lett. 75, 1226 (1995).

[11] A. Y. Rednikov, M. G. Velarde, and Y. S. Ryazantsev, Active drops and drop motions due to nonequilibrium phenomena, J. Non-Equilib. Thermodyn. 19, 95 (1994).

[12] S. Soh, K. J. M. Bishop, and B. A. Grzybowski, Dynamic selfassembly in ensembles of camphor boats, J. Phys. Chem. B 112, 10848 (2008).

[13] C. C. Maass, C. Krüger, S. Herminghaus, and C. Bahr, Swimming droplets, Annu. Rev. Condens. Matter Phys. 7, 171 (2016).

[14] S. Michelin, E. Lauga, and D. Bartolo, Spontaneous autophoretic motion of isotropic particles, Phys. Fluids 25, 061701 (2013).
[15] D. Boniface, C. Cottin-Bizonne, R. Kervil, C. Ybert, and F. Detcheverry, Self-propulsion of symmetric chemically active particles: Point-source model and experiments on camphor disks, Phys. Rev. E 99, 062605 (2019).

[16] Y. Hayashima, M. Nagayama, and S. Nakata, A camphor grain oscillates while breaking symmetry, J. Phys. Chem. B 105, 5353 (2001).

[17] M. Nagayama, S. Nakata, Y. Doi, and Y. Hayashima, A theoretical and experimental study on the unidirectional motion of a camphor disk, Physica D 194, 151 (2004).

[18] J. Toner, Y. Tu, and S. Ramaswamy, Hydrodynamics and phases of flocks, Ann. Phys. (NY) 318, 170 (2005).

[19] M. E. Cates and J. Tailleur, Motility-induced phase separation, Annu. Rev. Condens. Matter Phys. 6, 219 (2015).

[20] H. Ender, A. K. Froin, H. Rehage, and J. Kierfeld, Surfactantloaded capsules as Marangoni microswimmers at the air-water interface: Symmetry breaking and spontaneous propulsion by surfactant diffusion and advection, Eur. Phys. J. E 44, 21 (2021).

[21] H. Ender and J. Kierfeld, From diffusive mass transfer in Stokes flow to low Reynolds number Marangoni boats, Eur. Phys. J. E 44, 4 (2021).

[22] Y. Koyano, T. Sakurai, and H. Kitahata, Oscillatory motion of a camphor grain in a one-dimensional finite region, Phys. Rev. E 94, 042215 (2016).

[23] H. Kitahata, S.-I. Hiromatsu, Y. Doi, S. Nakata, and M. R. Islam, Self-motion of a camphor disk coupled with convection, Phys. Chem. Chem. Phys. 6, 2409 (2004).

[24] Note that, experimentally, the sink term ascribed to sublimation can also be provided by solubilization in the subphase.

[25] N. J. Suematsu, Y. Ikura, M. Nagayama, H. Kitahata, N. Kawagishi, M. Murakami, and S. Nakata, Mode-switching of the self-motion of a camphor boat depending on the diffusion distance of camphor molecules, J. Phys. Chem. C 114, 9876 (2010).

[26] N. J. Suematsu, T. Sasaki, S. Nakata, and H. Kitahata, Quantitative estimation of the parameters for self-motion driven by difference in surface tension, Langmuir 30, 8101 (2014).

[27] V. Akella, D. K. Singh, S. Mandre, and M. Bandi, Dynamics of a camphoric acid boat at the air-water interface, Phys. Lett. A 382, 1176 (2018).

[28] H. Kitahata and N. Yoshinaga, Effective diffusion coefficient including the Marangoni effect, J. Chem. Phys. 148, 134906 (2018).

[29] T. Bickel, Spreading dynamics of reactive surfactants driven by marangoni convection, Soft Matter 15, 3644 (2019). 
[30] M. R. Maxey and J. J. Riley, Equation of motion for a small rigid sphere in a nonuniform flow, Phys. Fluids 26, 883 (1983).

[31] R. Gatignol, The faxen formulae for a rigid particle in an unsteady non-uniform stokes flow, J. Mécanique Théorique et Appliquée 2, 143 (1983).

[32] T. M. Burton and J. K. Eaton, Fully resolved simulations of particle-turbulence interaction, J. Fluid Mech. 545, 67 (2005).

[33] R. Volk, E. Calzavarini, G. Verhille, D. Lohse, N. Mordant, J. F. Pinton, and F. Toschi, Acceleration of heavy and light particles in turbulence: comparison between experiments and direct numerical simulations, Physica D 237, 2084 (2008).

[34] E. Calzavarini, R. Volk, M. Bourgoin, E. Leveque, J. F. Pinton, and F. Toschi, Acceleration statistics of finite-sized particles in turbulent flow: the role of Faxen forces, J. Fluid Mech. 630, 179 (2009).

[35] E. Calzavarini, R. Volk, E. Lévêque, J.-F. Pinton, and F. Toschi, Impact of trailing wake drag on the statistical properties and dynamics of finite-sized particle in turbulence, Physica D 241, 237 (2012), Special Issue on Small Scale Turbulence.

[36] N. M. Qureshi, M. Bourgoin, C. Baudet, A. Cartellier, and Y. Gagne, Turbulent Transport of Material Particles: An Experimental Study of Finite Size Effects, Phys. Rev. Lett. 99, 184502 (2007).

[37] R. D. Brown, Z. Warhaft, and G. A. Voth, Acceleration Statistics of Neutrally Buoyant Spherical Particles in Intense Turbulence, Phys. Rev. Lett. 103, 194501 (2009).

[38] R. Volk, E. Calzavarini, E. Leveque, and J.-F. Pinton, Dynamics of inertial particles in a turbulent von Karman flow, J. Fluid Mech. 668, 223 (2011).

[39] R. Volk, C. Mauger, M. Bourgoin, C. Cottin-Bizonne, C. Ybert, and F. Raynal, Chaotic mixing in effective compressible flows, Phys. Rev. E 90, 013027 (2014).

[40] C. Gouiller, F. Raynal, L. Maquet, M. Bourgoin, C. CottinBizonne, R. Volk, and C. Ybert, Mixing and unmixing induced by active camphor particles, Phys. Rev. Fluids 6, 014501 (2021).

[41] J. Denk and E. Frey, Pattern-induced local symmetry breaking in active-matter systems, Proc. Natl. Acad. Sci. 117, 31623 (2020).

[42] S. Nakata, Y. Doi, and H. Kitahata, Synchronized Sailing of Two Camphor Boats in Polygonal Chambers, J. Phys. Chem. B 109, 1798 (2005).

[43] Y. Hirose, Y. Koyano, and M. Nagayama, Two floating camphor particles interacting through lateral capillary force, J. Phys. Soc. Jpn. 89, 074004 (2020).

[44] K. Lippera, M. Benzaquen, and S. Michelin, Bouncing, chasing, or pausing: Asymmetric collisions of active droplets, Phys. Rev. Fluids 5, 032201(R) (2020).

[45] K. Lippera, M. Morozov, M. Benzaquen, and S. Michelin, Collisions and rebounds of chemically active droplets, J. Fluid Mech. 886, 1843 (2020).

[46] K. Lippera, M. Benzaquen, and S. Michelin, Alignment and scattering of colliding active droplets, Soft Matter 17, 365 (2021).

[47] P. G. Moerman, H. W. Moyses, E. B. van der Wee, D. G. Grier, A. van Blaaderen, W. K. Kegel, J. Groenewold, and J. Brujic, Solute-mediated interactions between active droplets, Phys. Rev. E 96, 032607 (2017).

[48] B. V. Hokmabad, S. Saha, J. Agudo-Canalejo, R. Golestanian, and C. C. Maass, Quantitative characterization of chemore- pulsive alignment-induced interactions in active emulsions, arXiv:2012.05170.

[49] P. Romanczuk, M. Bär, W. Ebeling, B. Lindner, and L. Schimansky-Geier, Active brownian particles, Eur. Phys. J.: Spec. Top. 202, 1 (2012).

[50] Y. S. Ikura, E. Heisler, A. Awazu, H. Nishimori, and S. Nakata, Collective motion of symmetric camphor papers in an annular water channel, Phys. Rev. E 88, 012911 (2013).

[51] N. J. Suematsu, K. Tateno, S. Nakata, and H. Nishimori, Synchronized intermittent motion induced by the interaction between camphor disks, J. Phys. Soc. Jpn. 84, 034802 (2015).

[52] H. Masoud and M. J. Shelley, Collective Surfing of Chemically Active Particles, Phys. Rev. Lett. 112, 128304 (2014).

[53] A. Domínguez, P. Malgaretti, M. N. Popescu, and S. Dietrich, Collective dynamics of chemically active particles trapped at a fluid interface, Soft Matter 12, 8398 (2016).

[54] A. Domínguez and M. N. Popescu, Phase coexistence in a monolayer of active particles induced by Marangoni flows, Soft Matter 14, 8017 (2018).

[55] S. Thutupalli, R. Seemann, and S. Herminghaus, Swarming behavior of simple model squirmers, New J. Phys. 13, 073021 (2011).

[56] C. Krüger, C. Bahr, S. Herminghaus, and C. C. Maass, Dimensionality matters in the collective behaviour of active emulsions., Eur. Phys. J. E: Soft. Matter 39, 64 (2016).

[57] N. Yoshinaga, Simple models of self-propelled colloids and liquid drops: From individual motion to collective behaviors, J. Phys. Soc. Jpn. 86, 101009 (2017).

[58] J. R. Howse, R. A. L. Jones, A. J. Ryan, T. Gough, R. Vafabakhsh, and R. Golestanian, Self-Motile Colloidal Particles: From Directed Propulsion to Random Walk, Phys. Rev. Lett. 99, 048102 (2007).

[59] J. Palacci, C. Cottin-Bizonne, C. Ybert, and L. Bocquet, Sedimentation and Effective Temperature of Active Colloidal Suspensions, Phys. Rev. Lett. 105, 088304 (2010).

[60] E. R. Weeks, Three-dimensional direct imaging of structural relaxation near the colloidal glass transition, Science 287, 627 (2000).

[61] N. Klongvessa, F. Ginot, C. Ybert, C. Cottin-Bizonne, and M. Leocmach, Nonmonotonic behavior in dense assemblies of active colloids, Phys. Rev. E 100, 062603 (2019).

[62] D. R. Nelson and B. I. Halperin, Dislocation-mediated melting in two dimensions, Phys. Rev. B 19, 2457 (1979).

[63] A. L. Thorneywork, J. L. Abbott, D. G. Aarts, P. Keim, and R. P. Dullens, Bond-orientational order and frank's constant in twodimensional colloidal hard spheres, J. Phys.: Condens. Matter 30, 104003 (2018).

[64] K. Zahn and G. Maret, Dynamic Criteria for Melting in Two Dimensions, Phys. Rev. Lett. 85, 3656 (2000).

[65] P. Digregorio, D. Levis, A. Suma, L. F. Cugliandolo, G. Gonnella, and I. Pagonabarraga, Full Phase Diagram of Active Brownian Disks: From Melting to Motility-Induced Phase Separation, Phys. Rev. Lett. 121, 098003 (2018).

[66] B. Kadoch, D. Kolomenskiy, P. Angot, and K. Schneider, A volume penalization method for incompressible flows and scalar advection, diffusion with moving boundaries, J. Comput. Phys. 231, 4365 (2012).

[67] C. Barentin, C. Ybert, J.-M. Di Meglio, and J.-F. Joanny, Surface shear viscosity of gibbs and langmuir monolayers, J. Fluid Mech. 397, 331 (1999). 